\title{
Metastable states in glassy systems
}

\author{
Giulio Biroli \\ Laboratoire de Physique Théorique de l'Ecole Normale Supérieure*, \\ 24 rue Lhomond, 75231 Paris cedex 05, France. \\ Jorge Kurchan \\ P.M.M.H. Ecole Supérieure de Physique et Chimie Industrielles, \\ 10, rue Vauquelin, 75231 Paris CEDEX 05, France
}

(October 25, 2018)

\begin{abstract}
Truly stable metastable states are an artifact of the mean-field approximation or the zero temperature limit. If such appealing concepts in glass theory as configurational entropy are to have a meaning beyond these approximations, one needs to cast them in a form involving states with finite lifetimes. Starting from elementary examples and using results of Gaveau and Schulman, we propose a simple expression for the configurational entropy and revisit the question of taking flat averages over metastable states. The construction is applicable to finite dimensional systems, and we explicitly show that for simple mean-field glass models it recovers, justifies and generalises the known results. The calculation emphasises the appearance of new dynamical order parameters.
\end{abstract}

\section{INTRODUCTION}

Slowly relaxing systems such as glasses or compacting granular media can be viewed as having fast, local, quasiequilibrium dynamics, plus a slow, nonequilibrium drift. These two superposed motions can take different forms: 'cage' vibrations plus structural rearrangements in glasses; bulk fluctuations plus domain-wall motion in coarsening problems; etc. At a given long time, the fast motion covers a region of phase space which one may picture as a 'metastable state'.

Even though metastable states are a familiar and appealing concept, it turns out that defining them in an unambiguous manner in non-mean field models is quite subtle. This in turn has as a consequence that such standard ideas in glass theory as 'configurational entropy' (related to the number of metastable states) are not only hard to calculate, but are indeed, with the exception of some fortunate cases, approximate as concepts.

In this paper we show how these questions can be put on a well defined basis using a formalism [1] that does not rely on specifically mean-field concepts.

First of all: given that one can simulate and in certain cases calculate analytically the complete history of a sample starting from a quench, why should one have any need to introduce the apparently unneeded notion of 'metastable state'? Indeed, these states only come into play when one wishes to make arguments such as: 'phase space contains such and such a distribution of states, which will be accessed with such and such a probability by a typical dynamical history. Long-time out of equilibrium observables can be directly calculated by averaging the observables over some subset of states - and further reference to dynamics may be omitted'.

This kind of 'ergodic' argument was pioneered by Edwards [2], who proposed that in compacting or slowly flowing granular systems one can obtain the correct dynamical observables by averaging the values they take over all blocked configurations of a certain volume. It later turned out that mean-field glass models [3, 1 ] relaxing at zero temperature had exactly Edwards' ergodicity property [5]: at long times any nonequilibrium observable is correctly given by the typical value it takes over all local energy minima of the appropriate energy density.

A first problem arises when one wishes to apply this concept at finite temperatures (or vibration, in the case of granular media). There again, the mean-field case offers a suggestion: at non zero temperature Edwards' argument works as well, provided one substitutes 'energy minima' by 'free-energy minima' ('states'). This construction is possible because within mean-field we have a well defined notion of free-energy landscape, whose local minima are in some (but not all) cases related to completely stable distributions. However, as discussed by Franz and Virasoro [6], one needs to consider 'quasi-states' with finite lifetimes in order to understand the situation at finite waiting times.

*Unité Mixte de Recherche du Centre National de la Recherche Scientifique et de l'Ecole Normale Supérieure. 
In finite-dimensional problems, a high-lying metastable state cannot have an infinite lifetime: there is always a finite probability of escape through the nucleation of droplet of a more favourable phase. Hence, which distribution one considers as metastable depends always on which lifetimes one is considering. For example, the concept of 'configurational entropy' (the logarithm of the number of states), ubiquitous in glass theory, has in finite dimensions only a meaning with a timescale attached. Moreover, even the mere definition of an Edwards distribution is not as simple, quite apart from the question of the validity of the ergodicity-like hypothesis it assumes.

In section 2 we shall review the notion of metastable state within mean-field glass models and how the knowledge of their distribution allows in certain cases to reproduce some results obtained from the full solution of the out of equilibrium dynamics. We shall also mention some limitations found even at this level of the identification 'free-energy minimum stable state'.

In section 3 we discuss a strategy valid in any dimension for the definition and calculation of metastable states based on the evolution operator developed by Gaveau and Schulman [1]. We discuss how one can thus recast the question of configurational entropy and Edwards' distribution in a form relevant in finite dimensions at nonzero temperature (or stronger vibration, in the case of granular media) by considering finite-lifetime metastable states, in the spirit of the 'quasi-states' discussed in [6].

In section 4 we apply this method to a simple mean-field glass model. We show how one can rederive in this way both the number of states and the dynamics inside a state, within a framework whose applicability goes beyond mean-field.

\section{A FORTUNATE CASE: MEAN-FIELD MODELS}

Consider the mean-field model of ferromagnet:

$$
E=-\frac{1}{2 N} \sum_{i, j} S_{i} S_{j}-h \sum_{i} S_{i}
$$

where the sum is over all spins. The spins can be Ising $S_{i}= \pm 1$ or spherical $\sum_{i} S_{i}^{2}=N$. One can easily obtain a free energy in terms of local magnetisations $m_{i}=\left\langle S_{i}\right\rangle$ (where $\langle\cdot\rangle$ means the average over the Gibbs measure):

$$
f\left(\left\{m_{i}\right\}\right)=-\frac{1}{2 N} \sum_{i \neq j} m_{i} m_{j}-h \sum_{i} m_{i}-T S\left(\left\{m_{i}\right\}\right)
$$

where $T=1 / \beta$ denotes the temperature and $S\left(\left\{m_{i}\right\}\right)$ is the usual entropic term:

$$
\begin{aligned}
& S\left(\left\{m_{i}\right\}\right)=-\sum_{i} \frac{1}{2}\left[\left(1-m_{i}\right) \ln \left(1-m_{i}\right)+\left(1+m_{i}\right) \ln \left(1+m_{i}\right)\right] \quad \text { for } \quad S_{i}= \pm 1 \\
& =\frac{1}{2} \ln (1-q) \quad \text { spherical model }
\end{aligned}
$$

and $N q=\sum_{i=1}^{N} m_{i}^{2}$.

The states are represented by the minima of $f$. At $T<T_{c}$ there are two, and the deeper one dominates the Boltzmann distribution. If $h>0$ one of the states becomes metastable: within mean-field its lifetime is infinite, but in finite dimensions it will decay.

\section{The TAP approach}

Next, consider the glassy Hamiltonians:

$$
E=-\frac{1}{p !} \sum_{i_{1}, \ldots, i_{p}} J_{i_{1}, \ldots, i_{p}} S_{i_{1}} \ldots S_{i_{p}}
$$

where the $J_{i_{1}, \ldots, i_{p}}$ are independent random variables with variance $p ! / 2 N^{p-1}$. The Ising version with $p=2$ is the Sherrington-Kirkpatrick model, the mean field version of spin-glasses. The models with $p \geq 3$ are instead systems having the behaviour resembling structural glasses [7,8]. 
It turns out that one can find for these models a free energy function analogous to (2) (the 'TAP' free energy [9]), in terms of local magnetisations $m_{i}$ :

$$
f_{T A P}\left(\left\{m_{i}\right\}\right)=-\frac{1}{p !} \sum_{i_{1}, \ldots, i_{p}} J_{i_{1}, \ldots, i_{p}} m_{i_{1}} \ldots m_{i_{p}}-\frac{\beta}{4}\left[1-q^{p}-p q^{p-1}(1-q)\right]-T S\left(\left\{m_{i}\right\}\right)
$$

where $N q=\sum_{i} m_{i}^{2}$ and $S\left(\left\{m_{i}\right\}\right)$ is given in (3) for the spherical and Ising cases, respectively.

Within the TAP approach, one signature of the glass transition is the fact that the free energy (5) has, below a critical temperature, many $\left(\sim e^{a N}\right)$ minima [10, 11, 13, 12]. The main difference between mean-field versions of spin and structural glasses is seen, in the TAP approach, in the way states are separated.

Given the analogies with the ferromagnet, it seems very tempting to attribute to the minima of (5) a dynamical meaning of 'state'. For the models with $p \geq 3$ this has been done [14,15] by starting from a configuration where the coordinates are as close as possible to having a given $m_{i}^{a}$, and then checking that the subsequent dynamical evolution is a stable, quasi-equilibrium situation confined to a region of phase space in such a way that $\left\langle S_{i}\right\rangle_{\text {time-average }}=m_{i}^{a}$.

On the contrary, for models like the Sherrington-Kirkpatrick model the identification of all TAP-minima with stable states seems to breaks down. Let us formulate an heuristic argument to see this. Decreasing temperature, minima split in a second-order transition manner. At least a fraction of the minima are 'born' this way 16 , and to get an exponential number of minima one needs that on average there is a division every $O(1 / N)$ change in temperature. Hence, a fraction of TAP solutions are just $O(1 / N)$ below their critical temperature, and under those circumstances barriers cannot be large enough to dynamically separate them from their 'twins'.

\section{FDT-temperature. Long-time out of equilibrium observables}

The dynamics of model (何) following a quench below the critical temperature can be solved analytically [17, 1 . One finds that the system never equilibrates, and remains aging just above a threshold level of energy density $e_{t h}$ and free energy density $f_{\text {th }}$ higher than the equilibrium ones. Given any two observables $A$ and $B$, one can define their dynamic correlation function:

$$
C_{A B}\left(t, t^{\prime}\right)=\left\langle A(t) B\left(t^{\prime}\right)\right\rangle
$$

and the integrated response $\chi_{A B}$ to a field $h_{B}$ conjugate to $B$ acting between times $t^{\prime}$ and $t$ :

$$
\chi_{A B}\left(t, t^{\prime}\right)=\frac{\delta\langle A(t)\rangle}{\delta h_{B}}
$$

where the averages are over the dynamical realisation.

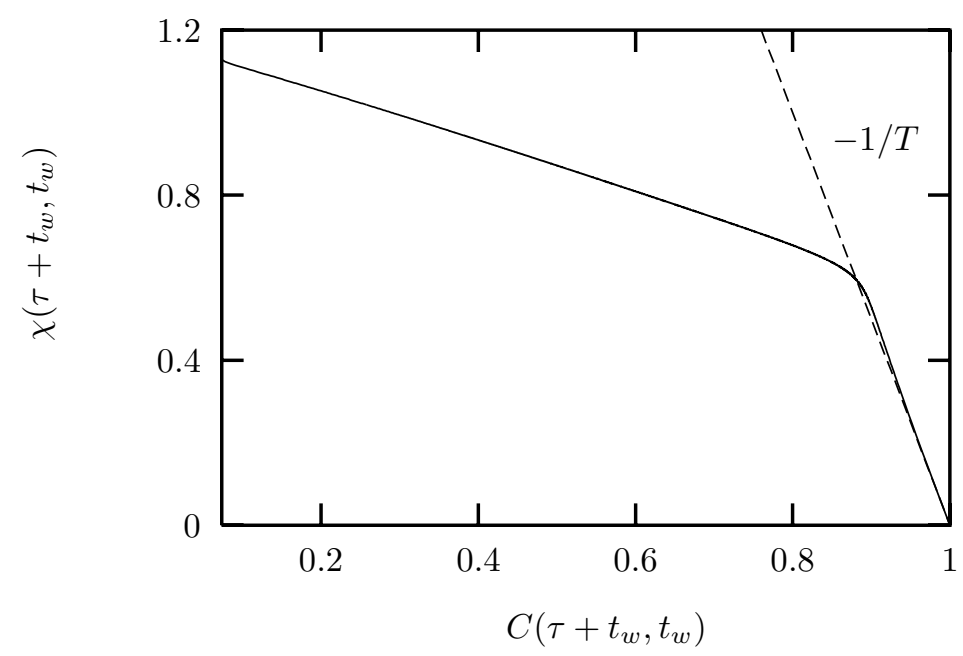

FIG. 1. A fluctuation-dissipation plot. The straight line to the left defines the effective temperature. 
If one makes a parametric plot of $\chi_{A B}\left(t, t^{\prime}\right)$ versus $C_{A B}\left(t, t^{\prime}\right)$ one obtains for model (14) with $p>2$ at long times a curve like Fig. 2. One has in addition to the straight line with gradient $-1 / T$ (as in equilibrium), another straight line of gradient, say, $-1 / T_{e f f}$, associated to the slow relaxation. $T_{\text {eff }}$ is the same for every pair of observables $A$ and $B$, and it can be shown [18] that it satisfies all the properties of a true temperature.

The appearance of a temperature in a system is an indication of some form of ergodicity, in this case clearly not the usual Gibbs-Boltzmann equilibrium at temperature $T$. Indeed, soon after the dynamical solution was obtained, Monasson and Virasoro 19,20 observed that the temperature $T_{\text {eff }}$ could be reobtained from the TAP approach without making any reference to the dynamics: Defining the complexity (or configurational entropy) $\mathcal{S}(f)$ as the logarithm of the number of TAP solutions of a given free energy, one checks that

$$
\frac{1}{T_{e f f}}=\left.\frac{\partial \mathcal{S}(f)}{\partial f}\right|_{f=f_{t h}}
$$

How this equality follows from Edwards' assumption is discussed in [6].

Furthermore, one can also see that the long-time values of macroscopic observables are given by the flat average of their values taken over all TAP solutions of the dynamical energy density $e_{t h}$. Hence, we have a strong indication for a measure à la Edwards, this time applied to TAP states.

If the system has large, but finite size, it will slowly approach equilibrium. In that case, a plot like Fig. 2 will show that the two tracts slowly tend to become parallel and $T_{\text {eff }}(t)$ (now a function of time) tends to $T$. Inspired by work of Bonilla et. al. [21, Nieuwenhuzen [22] conjectured that one could extend the relation (8) for all times, using the TAP solutions of the energy level appropriate at each time.

Later on, a two-temperature $\chi$ versus $C$ plot (and hence the existence of an FDT temperature $T_{\text {eff }}$ ) was seen to occur in realistic models such as three dimensional Lennard-Jones glasses [23 26]. Several of these simulations where performed at temperatures above the putative equilibrium glass temperature, so that the existence of a (slowly evolving) well-defined $T_{e f f}$ is surely a dynamical phenomenon, unrelated to the structure of equilibrium states. If one wishes to consider this as a symptom of slowly evolving flat distribution between metastable states, one finds oneself in the embarrassing situation that it is not entirely obvious what one means by 'metastable state' in finite dimensions, as we now discuss.

\section{The problem with finite dimensional and driven systems}

In finite dimensions and non-zero temperature nucleation arguments suggest that a distribution with dynamical free energy density (to be defined below) higher than the equilibrium one should decay through nucleation in finite times. We are hence in a situation in which we have no absolute notion of state without making reference to a timescale (and hence to dynamics): two different distributions may be confused into a single state or be treated as two separate entities depending on whether the time to go from one to the other is smaller than or larger than the timescale considered.

If we are interested in systems driven by shear or by vibration, we have the additional problem that even in the mean-field case the distribution is not Gibbsean within a state. In a vibrated case, the notion of stability must be substituted by the notion of periodicity, so that a 'state' will turn out to be a structure periodic in time.

Before entering into the present approach, let us mention that a pragmatic way of dealing with these difficulties, at least at very short time-scales, is the so-called 'inherent structure' construction [27]. Though it does not solve the questions of principle mentioned above [28], it offers a practical way around applicable to concrete problems.

\section{DYNAMICAL DEFINITION OF METASTABLE STATES}

Let us consider a system evolving with stochastic dynamics, which for definiteness we shall consider is of the Langevin form. The probability distribution will evolve according to:

$$
\begin{aligned}
\frac{d P(S, t)}{d t} & =-H P(S, t) \\
H & =-\frac{\partial}{\partial S_{i}}\left(T \frac{\partial}{\partial S_{i}}+\frac{\partial E}{\partial S_{i}}\right)
\end{aligned}
$$


where $H$ is the Fokker-Planck operator. The potential energy $E$ can be time-dependent, and furthermore one can add to $\left(\frac{\partial E}{\partial S_{i}}\right)$ forces that do not derive from a potential.

Given a probability distribution $P$, one can define a dynamic free energy

$$
F(t)=\int(T P(S, t) \ln P(S, t)+E(S) P(S, t)) d S
$$

If $H$ is time-independent, any stationary configuration satisfies

$$
H P_{\text {stationary }}=0
$$

Moreover, writing any distribution as $P(x, t)=\sum c_{i}(t) \psi_{i}(x)$ where $\psi_{i}(x)$ are the right eigenvectors of $H$ :

$$
H\left|\psi_{i}\right\rangle=\lambda_{i}\left|\psi_{i}\right\rangle
$$

the evolution equation (9) implies:

$$
c_{i}(t)=c_{i}^{o} e^{-\lambda_{i} t}
$$

We see that if $P$ is to vary slowly it has to be concentrated on eigenvectors with low eigenvalues $\lambda_{i}$. Indeed, each $\lambda_{i}$ is an inverse timescale [29, 30].

In the following subsections we shall motivate and discuss an identification of the set of small eigenvalue $\psi_{i}$ 's with metastable states.

\section{Motivation}

Consider first the system of two hard disks performing Langevin dynamics in a box (Fig. 2). Clearly, if the disks are really not interpenetrable, there are two different ergodic components, each composed of the mirror image of the configurations of the other. Symmetry implies that the spectrum of the Fokker-Planck operator is doubly degenerate. The two lowest (zero) eigenvalues correspond to two stationary distributions. One can construct an associated eigenvector as the flat distribution over all pairs of coordinates of the centers of the disks such that they do not superpose and such that the disk ' $\mathrm{A}$ ' is to the right and ' $\mathrm{B}$ ' to the left, and similarly a second eigenvector corresponding to having the disk ' $\mathrm{B}$ ' to the right and ' $\mathrm{A}$ ' to the left. These are the 'pure states': any linear combination of these two distributions will be an 'impure' state. The next higher eigenvalues are equal to the inverse of the time needed for the particles to explore their ergodic component. Note, in passing, that this hard-spheres system is the typical example in which the inherent structure construction is not meaningful while the present one has no problem.

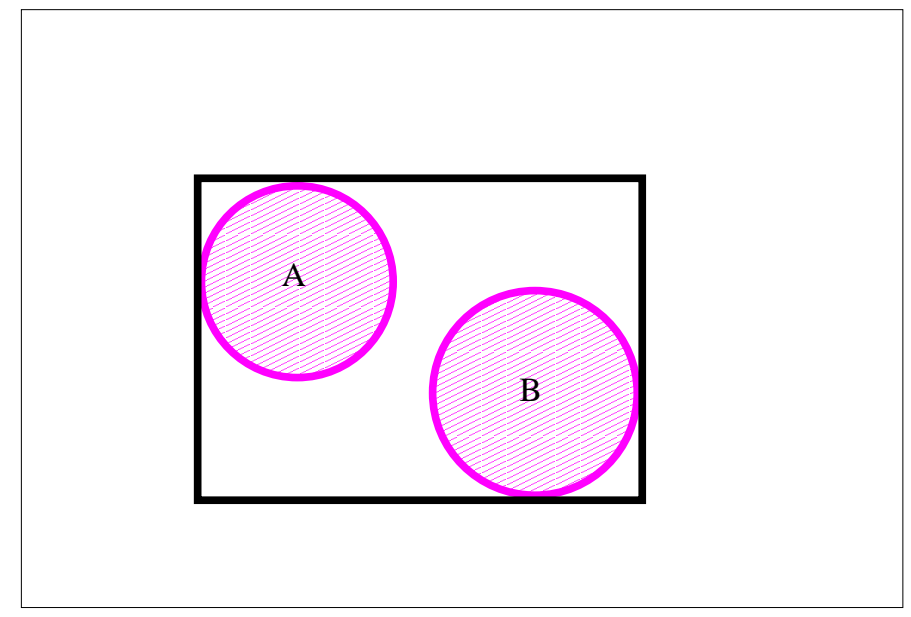

FIG. 2. A system with two ergodic components.

In this example we have strictly two ergodic components, corresponding to twofold ground state degeneracy of the Fokker-Planck operator. This is indeed very general: suppose we have $p$ ergodic components $\mathcal{C}_{1}, \ldots, \mathcal{C}_{p}$, with typical times $t_{1}, \ldots, t_{p}$ required to explore each component. We can construct an independent eigenvector with zero eigenvalue 
using the stationary distribution $P_{a}(x)$ restricted to each component $\mathcal{C}_{a}$. These completely span the zero eigenvalue subspace. To show this, we calculate:

$$
\begin{aligned}
\operatorname{tr}\left[e^{-t^{*} H}\right] & =\int d x \int d y\left\langle x\left|e^{-\frac{t^{*}}{2} H}\right| y\right\rangle\left\langle y\left|e^{-\frac{t^{*}}{2} H}\right| x\right\rangle=\sum_{a=1}^{p} \sum_{b=1}^{p} \int_{x \in \mathcal{C}_{a}} d x \int_{y \in \mathcal{C}_{b}} d y\left\langle x\left|e^{-\frac{t^{*}}{2} H}\right| y\right\rangle\left\langle y\left|e^{-\frac{t^{*}}{2} H}\right| x\right\rangle \\
& =\sum_{a=1}^{p} \int_{x \in \mathcal{C}_{a}} d x \int_{y \in \mathcal{C}_{a}} d y\left\langle x\left|e^{-\frac{t^{*}}{2} H}\right| y\right\rangle\left\langle y\left|e^{-\frac{t^{*}}{2} H}\right| x\right\rangle
\end{aligned}
$$

If we now take $t^{*}$ much larger than all the $t_{i},\left\langle y\left|e^{-\frac{t^{*}}{2} H}\right| x\right\rangle \simeq P_{a}(y)$, the equilibrium probability for $y$ restricted to the ergodic component $a$ to which $x$ belongs. Hence:

$$
\operatorname{tr}\left[e^{-t^{*} H}\right] \simeq \sum_{a=1}^{p} \sum_{b=1}^{p} \int_{x \in \mathcal{C}_{a}} d x \int_{y \in \mathcal{C}_{a}} d y P(x) P(y) \simeq p
$$

This shows that the number of states 'below the gap' coincides with the number of ergodic components.

In the preceding examples the ergodic components are strictly separated. However, in most applications this is not the case: there is in fact a passage time between components that only becomes infinite in some limit. To understand the construction in these cases, consider a very low temperature Langevin process occurring in asymmetric and symmetric double-well potentials as in Figure 3. On the left of the figure we show the lowest levels of the spectrum for both cases, and at the top the corresponding eigenvectors. For the asymmetric case, the two lowest eigenvalues are separated by the inverse Arrhenius escape time from the highest minimum. All other eigenvalues are much higher $(O(1))$, and include the escape time from a maximum, etc. The eigenfunction labeled a is essentially positive and represents a "pure" state $P_{1}$, while one can make a linear combination $P_{2}=(\mathbf{a}+\mathbf{b})$ that will also be positive and concentrated on the metastable minimum. For the symmetric well the situation is similar, but now it is the linear combinations $P_{1}=(\mathbf{a}+\mathbf{b})$ and $P_{2}=(\mathbf{a}-\mathbf{b})$ that play the role of "pure" states. Any other combination of the form $y P_{1}+(1-y) P_{2}$ with $0<y<1$ will give an 'impure' (almost) steady state.

Note that these definitions make sense in the time-window in which we can consider the exponential Arrhenius times much larger that any other time involved $(O(1))$, and this will happen only in the low temperature limit.

If the temperature is non-zero, a separation of timescales can happen as a result of the thermodynamical (or other) limit. For example, it is easy to see that a mean-field ferromagnet at $0<T<T_{c}$ will have a similar spectrum, but with $N$ playing the role of large parameter instead of $1 / T$. The case of finite dimensional ferromagnets is slightly more subtle: we have there a timescale for domain excitations within a state that can be as large as a power law in $N$ (the time it takes for a large domain to collapse), and a much longer timescale $\left(\sim e^{c N^{(d-1) / d}}\right)$ for going from one phase to the other.

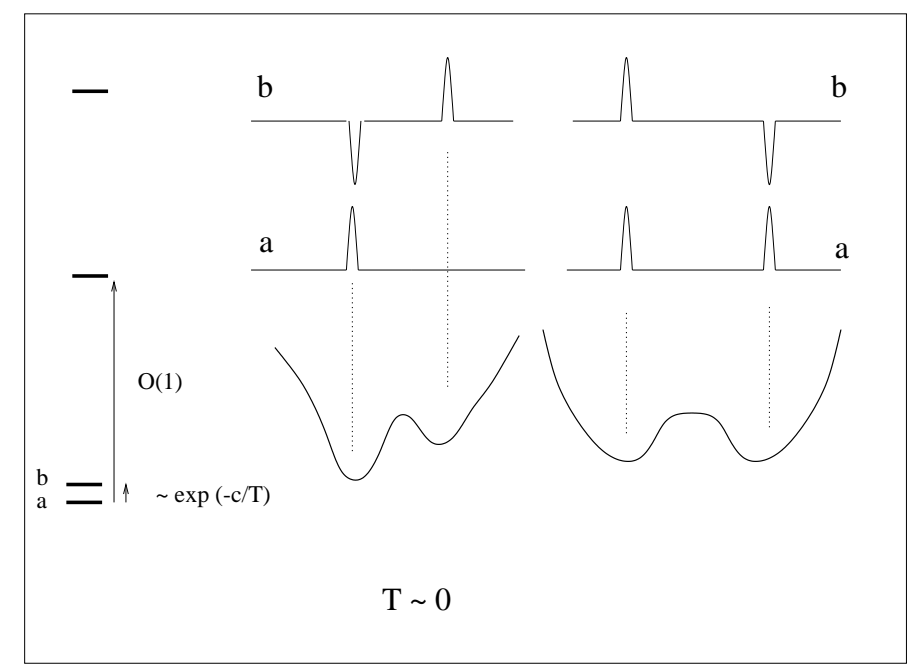

FIG. 3. A sketch of eigenfunctions and spectrum of the Fokker-Planck operator at low temperature .

The construction of Gaveau and Schulman 
In general, the low eigenvalue spectrum can correspond to more than two eigenvectors. One can now ask in general whether all these eigenvectors (or combinations of them) represent positive, stable distributions, and whether one can construct as many pure states as there are low eigenvalues. In a series of papers [1], Gaveau, Schulman and Lesne showed this to be the case, gave recipes for the explicit construction of the pure states, proved their unicity and exploited this construction to study metastability.

We shall be only descriptive here, we refer the reader to the references for the proofs, as well as other investigations concerning metastability. Consider for example a Fokker-Planck operator having the lowest $p$ eigenvalues $\lambda_{1}, \ldots, \lambda_{p}$ separated by a gap from the others, 1.e. one can find a $t^{*}$ such that one can consider that:

$$
\begin{array}{ll}
t^{*} \lambda_{i}<<1 & \text { for } \quad i=1, \ldots, p \\
t^{*} \lambda_{i}>>1 & \text { for } \quad i=p+1, \ldots
\end{array}
$$

In the previous simple example any $t^{*}$ such that $t^{*}$ is positive will satisfy this as $T \rightarrow 0$. The meaning of $t^{*}$ is clear: it is a timescale much longer than the relaxation into states, but much shorter than transitions between states. Clearly, the operator $\exp \left[-t^{*} H\right]$ is essentially a projector onto the space 'below the gap' (up to terms of order exp[- $\left.t^{*} \lambda_{i}\right]$, with $i \leq p)$. Within the same accuracy, one can then find a basis of $p$ right eigenvectors $\left|P_{i}\right\rangle$ which are:

- positive: $P_{i}(x)=\left\langle x \mid P_{i}\right\rangle \geq 0$ :

- almost stationary: $H\left|P_{i}\right\rangle \sim 0 \quad \forall i=1, \ldots, p$

- normalised and not zero in non-overlapping regions of space.

The last property is related to the fact that one can also find a basis of $p$ approximate almost stationary $\left(\left\langle Q_{i}\right| H \sim 0\right)$ left eigenvectors $\left\langle Q_{i}\right|$, such that each $Q_{i}$ is essentially one within the support of $P_{i}(x)$, zero everywhere else and satisfy the orthogonality and normalisation conditions:

$$
\left\langle Q_{i} \mid P_{j}\right\rangle \sim \delta_{i j}
$$

Given any observable $A$, we can calculate its average within the state " $i$ " as:

$$
\langle A\rangle_{i}=\left\langle Q_{i}|A| P_{i}\right\rangle
$$

One can also write approximately:

$$
e^{-t^{*} H} \sim \sum_{i}\left|P_{i}\right\rangle\left\langle Q_{i}\right|
$$

As a consequence the $\left|P_{i}\right\rangle$ vectors have all the good properties to represents metastable states: they are positive normalised distributions, non zero only on different regions of the configuration space and they are stationary on time scales less than $t^{*}$. In the proof, as in the simple example of the previous subsection, the definition is unavoidably linked to a timescale: if one considers really infinite times, before any other limit, then the distinction between states vanishes. Furthermore, it is assumed the number of states so defined remains finite in the thermodynamic limit. We believe that going to situations in which this is not the case (as we will below) is indeed not entirely innocent, but is at the heart of quite a few problems associated to the definition of "state" in glassy systems. (We have already encountered such subtleties when we discussed TAP minima in the Sherrington-Kirkpatrick model).

\section{Driven systems.}

The construction described above is not limited to purely relaxational or time-independent systems. Consider for example the case in which a system is periodically 'vibrated' or 'tapped'. One can still try to look for stationary, or rather, periodic situations. One can repeat essentially the same argument by considering the evolution operator through one cycle:

$$
U=\mathcal{T} \int_{\text {cycle }} d t e^{-t H(t)}
$$

where $\mathcal{T}$ denotes time-order. One has to look now for the eigenvectors of $U$ whose eigenvalue is close to one. 
Similarly, one can also work with systems driven by constant nonconservative forces (as in a sheared fluid), and with nonlinear space-dependent friction (as in granular systems).

\section{Pure barriers.}

Given that pure states can be viewed as playing the role for finite temperature that certain energy minima play for zero temperature, one is naturally led to ask which distributions play the role of barriers (or in general saddle points) in finite temperature. For Fokker-Planck processes this can be done naturally starting from the states constructed as above. In Appendix A we show how this can be done.

We remark that a solvable example, in which saddle points play an important role in the spectrum of the evolution operators, is the Glauber evolution of the completely connected Ising model and its generalizations [30]. It has been found that the lowest eigenvalues, i.e. the longest relaxation times, are gathered in families, each one being in correspondence with a stationary, not necessary stable, point of the static mean field free energy.

\section{FLAT DISTRIBUTION OVER STATES AT FINITE TEMPERATURE OR VIBRATION. A DEFINITION OF CONFIGURATIONAL ENTROPY.}

\section{Motivation.}

As we mentioned in Section II, the fact that one finds a two-temperature behaviour in mean-field glasses can be seen as suggesting the relevance of a measure consisting in summing over metastable states of given energy (or free energy) with equal weight. Armed with the construction for metastable states we discussed above, we shall see how this measure can be expressed in finite dimensional, or periodically driven systems.

\section{Expressions.}

Consider, in the spirit of Edwards' distribution, an average of an observable over states, each measured with equal weight. This will be relevant for the long-time out of equilibrium dynamics under the assumption that almost all states of given energy have the same basin of attraction.

Using the same notation as in the previous section, we define the average over the measure $\langle\langle\cdot\rangle\rangle$ of an observable A in the following way:

$$
\langle\langle A\rangle\rangle_{t^{*}}=\frac{1}{p} \sum_{i=1}^{p}\langle A\rangle_{i}
$$

where the subindex $t^{*}$ reminds us of the fact that states are now defined according to their timescale. We have:

$$
\sum_{i=1}^{p}\langle A\rangle_{i}=\sum_{i=1}^{p}\left\langle Q_{i}|A| P_{i}\right\rangle=\sum_{i=1}^{p} \operatorname{tr}\left[\left|P_{i}\right\rangle\left\langle Q_{i}\right| A\right]=\operatorname{tr}\left[e^{-t^{*} H} A\right]
$$

where we have used (18) and (19). Hence:

$$
\langle\langle A\rangle\rangle_{t^{*}}=\frac{\operatorname{tr}\left[e^{-t^{*} H} A\right]}{\operatorname{tr}\left[e^{-t^{*} H}\right]}
$$

Note that once written this way, all reference to pure states has disappeared, except indirectly in the value chosen for $t^{*}$.

We often need an equation like (23), but restricted to states having a certain energy, particle number, etc. In that case we generalise (23) as, for example:

$$
\langle\langle A\rangle\rangle_{t^{*}}\left(E_{o}\right)=\frac{\operatorname{tr}\left[\delta\left(E-E_{o}\right) e^{-t^{*} H} A\right]}{\operatorname{tr}\left[\delta\left(E-E_{o}\right) e^{-t^{*} H}\right]}=\frac{\operatorname{tr}_{E_{o}}\left[e^{-t^{*} H} A\right]}{\operatorname{tr}_{E_{o}}\left[e^{-t^{*} H}\right]}
$$


where $\operatorname{tr}_{E_{o}}$ denotes a restricted trace.

Once we make the assumption that all states of the same energy (or particle number, etc) have an equal weight for the purposes of calculating a dynamical observable, it becomes meaningful to count their number at given energy, the configurational entropy:

$$
\mathcal{S}_{t^{*}}\left(E_{o}\right) \equiv \ln \operatorname{tr}\left[\delta\left(E-E_{o}\right) e^{-t^{*} H}\right]=\ln \operatorname{tr}_{E_{o}}\left[e^{-t^{*} H}\right]
$$

If $t^{*} \rightarrow 0$ we get the microcanonical measure (and entropy, up to irrelevant constants 31 ), and if we let $t^{*} \rightarrow \infty$ we find no high-lying metastable states at all in finite dimensions. The dependence on $t^{*}$ is hence unavoidable if one is to obtain a finite configurational entropy in that case.

Equation (25) defines the timescale-dependent configurational entropy. One also needs the average entropy within a state $s_{t^{*}}$, and the corresponding average free-energy of a state $f_{t^{*}}=E_{o}-T s_{t^{*}}$. Using the construction of (17), (18) and (19), we have:

$$
s_{t^{*}}\left(E_{o}\right)=-\frac{\int_{x / E(x)=E_{o}} d x\left\langle x\left|e^{-t^{*} H}\right| x\right\rangle \ln \left[\left\langle x\left|e^{-t^{*} H}\right| x\right\rangle\right]}{\int_{x^{\prime} / E\left(x^{\prime}\right)=E_{o}} d x^{\prime}\left\langle x^{\prime}\left|e^{-t^{*} H}\right| x^{\prime}\right\rangle}
$$

The meaning of this equation becomes transparent in the example of the completely separated ergodic components of the previous section.

Note that also the intra-state entropy is timescale-dependent. Indeed, if we set $t^{*} \rightarrow 0$, we are defining as 'states' the configurations themselves [31]. On the other extreme, if $t^{*}$ is longer than the equilibration time, $\left\langle x^{\prime}\left|e^{-t^{*} H}\right| x^{\prime}\right\rangle$ gives the Gibbs-measure, and the intra-state entropy becomes the usual entropy. In short: changing the timescale both changes the number of states and their nature, hence the change in configurational and intra-state entropy, respectively.

\section{Flat measures and effective temperatures}

Suppose we have a glassy system, taken through a given thermal history (an annealing protocol) to a glassy phase at time $t$, at which time its energy is $E(t)=E_{o}$ (and if we let the particle number or the volume change we should specify also them). The assumption of typicality of metastable states, is then:

$$
\langle\langle A\rangle\rangle_{t^{*}}\left(E_{o}\right)=\frac{\operatorname{tr}_{E_{o}}\left[e^{-t^{*} H} A\right]}{\operatorname{tr}_{E_{o}}\left[e^{-t^{*} H}\right]} \sim\langle A\rangle_{\text {history }}
$$

where the average is over several realisations of the same protocol, ending at time $t$ with energy $E(t)=E_{o}$.

From the Langevin point of view, the left hand side of (27) corresponds to adding over all periodic trajectories starting from an energy $E_{o}$ with period $t^{*}$. Equation (27) tells us that thermal histories give the same result as a very particular set of trajectories, in a manner analogous as when one represents chaotic dynamical systems by using only the periodic orbits.

In the zero-temperature or in the mean-field case, we can set $t^{*}=\infty$ in (27), and thus select the states with infinite lifetime. This is Edwards' prescription for granular media $(T \sim 0)$ and the one we discussed above within mean-field. In finite dimensions, where metastable states eventually nucleate, we are forced to give $t^{*}$ a finite value. The fact that the choice of $t^{*}$ is not unique already tells us that equation (27) will be an approximation.

The central remaining question now is what is a reasonable value for $t^{*}$. Indeed, giving a value of $t^{*}$ determines a configurational entropy $\mathcal{S}_{t^{*}}\left(E_{o}\right)$ and an intra-state free-energy: $f_{t^{*}}\left(E_{o}\right)=E_{o}-T s_{t^{*}}\left(E_{o}\right)$. This in turn determines a temperature associated with the timescale as:

$$
T_{t^{*}} \equiv\left[\frac{\partial \mathcal{S}_{t^{*}}}{\partial f_{t^{*}}}\right]^{-1}
$$

(where we have eliminated the energy in favour of $f_{t^{*}}$ ). It seems now natural to compare the different relaxation times of the correlations in a given problem with $t^{*}$. For example, glassy systems can often be described with two timescales, a fast (' $\beta$ ') relaxation and a slow, (waiting time dependent if the system is aging) ' $\alpha$-relaxation' $t_{\alpha}$. If $t^{*}$ is small $\left(t^{*}<<t_{\beta}\right)$, Eqn. (28) gives $T_{\text {eff }} \sim T$ [31]. If we put instead:

$$
t_{\beta}<<t^{*} \lesssim t_{\alpha}
$$


then $T_{t^{*}}$ may be different (larger) than $T$. This is the temperature to compare with the one governing the relation between correlation and response in the regime corresponding to the $\alpha$-relaxation [32].

There are cases in which the 'slow' relaxations happen in several timescales, becoming more and more different as aging proceeds (1. Then, the definition (28) immediately yields a different temperature for every widely separated timescale, and this would agree with what one observes from fluctuation-dissipation relations [4].

\section{Time dependent order parameters}

We are interested in calculating two-time correlation functions:

$$
<A(t) B\left(t^{\prime}\right)>\equiv \frac{1}{\mathcal{N}} \operatorname{tr}\left[e^{-\left(t^{*}-t\right) H} A e^{-\left(t-t^{\prime}\right) H} B e^{-t^{\prime} H}\right]=\frac{1}{\mathcal{N}} \operatorname{tr}\left[e^{-\left(t^{*}-\tau\right) H} A e^{-\tau H} B\right]=<A B>(\tau)
$$

where $\tau=t-t^{\prime}$, and $\mathcal{N}$ is the normalisation. Cyclic permutation implies:

$$
<A B>(\tau)=<B A>\left(t^{*}-\tau\right)
$$

If $H$ is a time-dependent Fokker-Planck operator, associated with forces deriving from a potential $E$, we have:

$$
e^{\beta E} H e^{-\beta E}=H^{\dagger}
$$

Using (32) in (30) we get the time-reversal property:

$$
\left.<A B>(\tau)=t r^{*}\left[e^{-\left(t^{*}-\tau\right) H}\left(e^{\beta E} B e^{-\beta E}\right)^{\dagger} e^{-\tau H}\left(e^{\beta E} A e^{-\beta E}\right)^{\dagger}\right]\right]=<\tilde{B} \tilde{A}>^{*}(\tau)
$$

where $\tilde{A} \equiv\left(e^{\beta E} A e^{-\beta E}\right)^{\dagger}$ and $\tilde{B} \equiv\left(e^{\beta E} B e^{-\beta E}\right)^{\dagger}$

We shall need to consider the cases in which $A$ and $B$ are respectively $S_{i}$ and $\hat{S}_{i} \equiv-\frac{\partial}{\partial S_{i}}$. Let us define, for a system of $N$ degrees of freedom:

$$
\begin{aligned}
& C\left(t-t^{\prime}\right)=C(\tau)=\frac{1}{N} \sum_{i}<S_{i}(t) S_{i}\left(t^{\prime}\right)> \\
& R\left(t-t^{\prime}\right)=R(\tau)=\frac{1}{N} \sum_{i}<S_{i}(t) \hat{S}_{i}\left(t^{\prime}\right)> \\
& D\left(t-t^{\prime}\right)=D(\tau)=\frac{1}{N} \sum_{i}<\hat{S}_{i}(t) \hat{S}_{i}\left(t^{\prime}\right)>
\end{aligned}
$$

Because we are considering periodic trajectories, causality is violated. This means that neither $D(\tau)$ nor $R(\tau)$ for negative $\tau$ need to be zero. However, it is easy to show that if $\left(t^{*}-\tau\right)$ is larger than the thermalisation time (in which the system is projected to its Gibbs measure), then we recover $D(\tau)=0$ and $R(\tau) R(-\tau)=0$. The existence of solutions violating causality for $t^{*}$ large is then a symptom of large equilibration times, i.e. of glassiness.

Using the time-reversal equation (33), it is easy to derive a non-causal form of FDT, valid for any $t^{*}$ :

$$
\frac{\partial}{\partial t^{\prime}} C\left(t-t^{\prime}\right)=T\{R(\tau)-R(-\tau)\}
$$

The physical meaning of $D$, unlike that of $C$ and $R$, is unfamiliar. If we couple the states to a time-dependent, random magnetic 'pinning fields' 19 $h_{i}$ such that $\overline{h_{i}(t) h_{j}\left(t^{\prime}\right)}=F\left(t, t^{\prime}\right) \delta_{i j}$, the fields will make a change in the number of metastable states, and it is easy to show that:

$$
D\left(t, t^{\prime}\right)=\frac{1}{N} \frac{\delta}{\delta F\left(t, t^{\prime}\right)} \ln \left\{\operatorname{tr}\left[e^{-t^{*} H}\right]\right\}
$$

It is hence clear that systems with a finite number of states will have $D=0$ in the long-time limit. 


\section{THE CALCULATION}

In the following we apply the theory discussed above to a simple glassy system: the spherical version of model (4). These models are thought to be mean-field versions of structural glasses, we shall not deal in this paper with models corresponding to spin-glasses for which, as mentioned above, we do not expect the present computation of states to be in correspondence with the TAP-equation based calculations in the literature.

The trace of the Fokker-Planck operator, at a fixed energy density $E$, can be written as a functional integral 33] over the spin fields $S_{i}(t)$ and the response fields $\hat{S}_{i}(t)$ with periodic boundary conditions on $S_{i}(t)$. Once the average of the trace has been performed [34], the action depends on the fields $S_{i}(t)$ and $\hat{S}_{i}(t)$ through the two time functions $C\left(t, t^{\prime}\right), R\left(t, t^{\prime}\right), D\left(t, t^{\prime}\right)$ only. As a consequence one can integrate out the fields $S_{i}(t)$ and $\hat{S}_{i}(t)$ and get an effective action on two time functions:

$$
\begin{aligned}
S / N & =-\left.\int_{0}^{t^{*}} d t\left(\partial_{t} R\left(t, t^{\prime}\right)+\lambda R\left(t, t^{\prime}\right)-T D\left(t, t^{\prime}\right)\right)\right|_{t^{\prime}=t^{+}} \\
& +\frac{p}{4} \int_{0}^{t^{*}} d t d t^{\prime}\left(D\left(t, t^{\prime}\right) C^{p-1}\left(t, t^{\prime}\right)+(p-1) R\left(t, t^{\prime}\right) R\left(t^{\prime}, t\right) C^{p-2}\left(t, t^{\prime}\right)\right) \\
& -\frac{\hat{\lambda}}{2} \int_{0}^{t^{*}} d t(C(t, t)-1)+\frac{1}{2} \operatorname{Tr} \ln \mathrm{M}
\end{aligned}
$$

where the operator $M$ reads:

$$
M=\left(\begin{array}{ll}
R\left(t, t^{\prime}\right) & C\left(t, t^{\prime}\right) \\
D\left(t, t^{\prime}\right) & R\left(t^{\prime}, t\right)
\end{array}\right)
$$

Since we consider times of order one with respect to $\mathrm{N}$ the functional integral is dominated by a saddle point contribution. We shall obtain periodic dynamic solutions which, in the glassy phase ( $a$ ) break causality, (b) have non-zero action, (c) satisfy time-translational invariance, and (d) satisfy time-reversal and its consequence (35). Note that (a) and (b) are properties typical of instantons, while (c) and (d) are not. In the high temperature phase there is a periodic solution with zero action for long times corresponding essentially to the equilibrium dynamics.

The stationarity conditions on the action are equivalent to four equations on the two-time functions:

$$
\begin{aligned}
& C^{\prime}(\tau)=-\lambda C(\tau)+2 T R(-\tau)+\frac{p}{2} \int_{0}^{t^{*}} d t^{\prime \prime} C^{p-1}\left(t-t^{\prime \prime}\right) R\left(t^{\prime}-t^{\prime \prime}\right)+k \int_{0}^{t^{*}} R\left(t-t^{\prime \prime}\right) C^{p-2}\left(t-t^{\prime \prime}\right) C\left(t^{\prime \prime}-t^{\prime}\right) d t^{\prime \prime} \\
& R^{\prime}(\tau)=-\lambda R(\tau)+2 T D(\tau)+\frac{p}{2} \int_{0}^{t^{*}} d t^{\prime \prime} C^{p-1}\left(t-t^{\prime \prime}\right) D\left(t^{\prime}-t^{\prime \prime}\right)+k \int_{0}^{t^{*}} d t^{\prime \prime} C^{p-2}\left(t-t^{\prime \prime}\right) R\left(t-t^{\prime \prime}\right) R\left(t^{\prime \prime}-t^{\prime}\right)+\delta(\tau) \\
& R^{\prime}(\tau)=-\lambda R(\tau)+k \int_{0}^{t^{*}} d t^{\prime \prime} D\left(t^{\prime}-t^{\prime \prime}\right) C^{p-2}\left(t^{\prime}-t^{\prime \prime}\right) C\left(t-t^{\prime \prime}\right)+k \int_{0}^{t^{*}} d t^{\prime \prime} C^{p-2}\left(t^{\prime}-t^{\prime \prime}\right) R\left(t-t^{\prime \prime}\right) R\left(t^{\prime \prime}-t^{\prime}\right) \\
& \quad+k(p-2) \int_{0}^{t^{*}} d t^{\prime \prime} C^{p-3}\left(t^{\prime}-t^{\prime \prime}\right) R\left(t^{\prime}-t^{\prime \prime}\right) R\left(t^{\prime \prime}-t^{\prime}\right) C\left(t-t^{\prime \prime}\right)-\hat{\lambda} C\left(t-t^{\prime}\right)+\delta(\tau) \\
& -D^{\prime}(\tau)=-\lambda D(\tau)+k \int_{0}^{t^{*}} d t^{\prime \prime} D\left(t^{\prime}-t^{\prime \prime}\right) R\left(t^{\prime \prime}-t\right) C^{p-2}\left(t-t^{\prime \prime}\right)+k \int_{0}^{t^{*}} d t^{\prime \prime} D\left(t-t^{\prime \prime}\right) C^{p-2}\left(t-t^{\prime \prime}\right) R\left(t^{\prime \prime}-t^{\prime}\right) \\
& \quad+k(p-2) \int_{0}^{t^{*}} d t^{\prime \prime} R\left(t-t^{\prime \prime}\right) R\left(t^{\prime \prime}-t\right) R\left(t^{\prime \prime}-t^{\prime}\right) C^{p-3}\left(t-t^{\prime \prime}\right)-\hat{\lambda} R(\tau)
\end{aligned}
$$

where $k=\frac{p(p-1)}{2}$ and we make explicitly use of the time translation invariance $\tau=t-t^{\prime}$. The spherical condition fix the value of $\hat{\lambda}$, which can be obtained subtracting eq. (41) to eq. (40) for $\tau=0$ :

$$
\hat{\lambda}=(p-2)\left(p / 2 \int_{0}^{\tau} d t^{\prime \prime} C^{p-1}\left(t-t^{\prime \prime}\right) D\left(t-t^{\prime \prime}\right)+k \int_{0}^{\tau} d t^{\prime \prime} R\left(t-t^{\prime \prime}\right) R\left(t^{\prime \prime}-t\right) C^{p-2}\left(t-t^{\prime \prime}\right)\right)-2 T D(0)
$$


Moreover fixing the value of the energy $E$ gives an equation on the spherical multiplier $\lambda$ :

$$
p E=-\lambda+T\left(R\left(0^{+}\right)+R\left(0^{-}\right)\right)
$$

as a consequence $E$ and $\lambda$ are directly related. Using the FDT relation one can show that (39-42) reduce to a set of three independent equations on the functions: $C(\tau), R(\tau)+R(-\tau)$ and $D(\tau)$.

\section{A. Time-reversal, non-causal solutions}

Let us show that computing for very large $t^{*}$ the trace of the Fokker-Planck operator one can recover the the number of stable states, and the dynamics within these states. The number of stable states can be obtained by a pure static computation for the p-spin spherical model $(p>2)$ using the TAP equations [13].

For very large $t^{*}$ there are two possible behaviours for the two-time functions depending on the energy (and the model) we consider:

- if at the energy value considered there are stable states then the action evaluated in the solution has a well defined limit as $t^{*} \rightarrow \infty$. In this same limit, one expects that the two-time functions for finite $\tau$ describe the dynamics inside a stable state (as calculated previously with other methods [14]). A careful analysis of equations (39 42) allows one to show that the asymptotic forms of two-time quantities reads for $\tau<<t^{*}$ :

$$
\begin{aligned}
& C(\tau)=C_{c}(\tau)+\frac{1}{t^{*}} \hat{C}(\tau), \quad R(\tau)=R_{c}(\tau)+\frac{1}{t^{*}}\left(\hat{R}(\tau)+r-r_{c}\right), \\
& D(\tau)=\frac{1}{t^{*}} D_{o}(\tau)+\frac{1}{\left(t^{*}\right)^{2}} \hat{D}\left(\frac{\tau}{t^{*}}\right)
\end{aligned}
$$

The function $R_{c}(\tau)$ is causal, and for large $\tau$ (but small with respect to $t^{*}$ ) we have that $R_{c}(\tau) \rightarrow 0$ and $C_{c}(\tau) \rightarrow q$. Together $R_{c}$ and $C_{c}$ describe the relaxation within a state. All other functions are of order one when their arguments are of order one, and tend to zero when their argument is large. The corrections of order $1 / t^{*}$ are not subleading in the computation of the trace, as this involves integrals over a large interval $\left[0, t^{*}\right]$. The Edwards-Anderson parameter $q$ and $r-r_{c}$ are order one constants to be determined in what follows. Note that the scaling of $D$ implies that $\hat{\lambda}$ is of order $1 / t^{*}$. One can easily check that $C_{c}(\tau)$ and $R_{c}(\tau)$ satisfy the equations describing the equilibrium dynamics inside a state studied in [14]:

$$
\begin{aligned}
C_{c}^{\prime}(\tau) & =\lambda C_{c}(\tau)+p / 2 \int_{0}^{t^{*}} d t^{\prime \prime} C_{c}^{p-1}\left(t-t^{\prime \prime}\right) R_{c}\left(t^{\prime}-t^{\prime \prime}\right) \\
& +k \int_{0}^{\mathrm{L}} R_{c}\left(t-t^{\prime \prime}\right) C_{c}^{p-2}\left(t-t^{\prime \prime}\right) C_{c}\left(t^{\prime \prime}-t^{\prime}\right) d t^{\prime \prime}+p^{2} q^{p-1}\left(r-r_{c}\right) / 2
\end{aligned}
$$

$R_{c}(\tau)$ is causal and related to the correlation function through the FDT relation $R_{c}(\tau)=-1 / T C_{c}^{\prime}(\tau) \theta(\tau)$.

- if for the energy value considered there are no stable states then the behaviour of the two-time functions is similar to the previous one except that $D$ has a part of order of one for finite time $D_{o}$ and a part of order or $1 / t^{*}$ for $\tau \sim t^{*}$ :

$$
D(\tau)=D_{o}(\tau)+\frac{1}{\left(t^{*}\right)} \hat{D}\left(\frac{\tau}{t^{*}}\right)
$$

As a consequence $C(\tau)$ and $R(\tau)$ do not satisfy equations 'within a state', and one has to solve a set of three equations on $C, R$ and $D$ in which $R$ is not causal also for infinite times $\left(t^{*}\right)$.

In the rest of this subsection we consider an energy such that stable states exists and we compute the zero-frequency values of $C, R$ and $D$. Using the asymptotic form introduced before one finds: 


$$
\begin{aligned}
& \int_{0}^{t^{*}} C(\tau) d \tau=q t^{*}+O(1) \\
& \int_{0}^{t^{*}} R_{c}(\tau) d \tau=r_{c}=\frac{1-q}{T} \\
& \int_{0}^{t^{*}} R(\tau) d \tau=\int_{0}^{t^{*}} R_{c}(\tau)+r-r_{c}+O\left(1 / t^{*}\right)=r+O\left(1 / t^{*}\right) \\
& \int_{0}^{t^{*}} D(\tau) d \tau=\frac{d}{t^{*}}+O\left(1 /\left(t^{*}\right)^{2}\right)
\end{aligned}
$$

Moreover for large $t^{*}$ the relation (43) reduces to the usual one: $p E=-\lambda+T / 2+O\left(1 / t^{*}\right)$. Therefore we can consider $\lambda$ as a fixed parameter.

Integrating eq. (39) between 0 and $t^{*}$ and taking the leading order in $t^{*}$ we obtain:

$$
q\left(-\lambda+\frac{p}{2 T}(1-q) q^{p-2}+\frac{p}{2 T}\left(1-q^{p-1}\right)+\frac{p^{2}}{2}\left(r-r_{c}\right) q^{p-2}\right)=0
$$

Subtracting eq. (39) evaluated in $\tau=0$ to (52) we get the usual equation on $\lambda$ and $q$ :

$$
\lambda=\frac{T}{1-q}+\frac{p}{2 T}\left(1-q^{p-1}\right)
$$

Since the spherical multiplier is a parameter, this equation fixes the overlap. It is useful to write this equation in a way which is directly related to the static computation. Using the following notation:

$$
q^{p / 2-1} p \mathcal{E}=-\lambda+\frac{p}{2 T}\left(1-q^{p-1}\right)-\frac{p(p-1)}{2 T} q^{p-2}(1-q) \quad z=(1-q) q^{p / 2-1} / T
$$

where $\mathcal{E}$ corresponds to the zero-temperature or radial energy which appear in the static computation, one can rewrite (53) as the usual static equation on $q$ [13]

$$
1+p z \mathcal{E}+p(p-1) z^{2} / 2=0
$$

From equation (52) we find the value of $r$ :

$$
r=-\frac{2}{p} q^{1-p / 2} \mathcal{E}
$$

Integrating (40) between 0 and $t^{*}$ and using eq. (52) we get the value of $d$ :

$$
q^{p-1} d=-2 / p+4 \mathcal{E}^{2} / p^{2}
$$

Finally we confirm, using the relationship between $\lambda$ and $q$, that the equations on $C_{c}(\tau)$ and $R_{c}(\tau)(46)$ are indeed the same ones found in [14] for the relaxational dynamics inside the stable state with overlap $q$ and energy $E=$ $(-\lambda+T / 2) / p$.

\section{B. The configurational entropy}

To obtain the number of stable states we have to inject the solution of eqs. (39-42) into the action (37) and then take the long time limit. Using the compact notation $Q\left(\tau ; t^{*}\right)=\left(C\left(\tau ; t^{*}\right), R\left(\tau ; t^{*}\right), D\left(\tau ; t^{*}\right)\right)$ for the set of the two-time functions, we decompose the asymptotic solution as $Q\left(\tau ; t^{*}\right)=Q_{0}\left(\tau ; t^{*}\right)+Q_{1}\left(\tau ; t^{*}\right) / t^{*}$, where $Q_{0}$ reads:

$$
Q_{0}\left(\tau ; t^{*}\right)=\left(C_{c}(\tau), R_{c}(\tau)+\frac{r-r_{c}}{t^{*}}, \frac{d}{\left(t^{*}\right)^{2}}\right)
$$

and $C_{c}(\tau), R_{c}(\tau)$ are the solution of the relaxational dynamics inside a stable state $(46)$ with the values of $r, r_{c}, d$ determined above. Using this decomposition and the fact that $Q$ is a saddle point of (37) we find for very large $t^{*}$ : 


$$
S(Q) \simeq S\left(Q_{0}\right)-\frac{1}{2} \frac{Q_{1}}{t^{*}} \otimes \frac{\delta^{2} S}{\delta Q^{2}} \otimes \frac{Q_{1}}{t^{*}}+\ldots \quad t^{*}>>1
$$

An explicit computation shows that the second term of (59) vanishes in the long time limit provided that the corrections to $q$ are of an order less than $1 / t^{*}$. This seems natural to us since we are considering the relaxation dynamics inside a stable state. As a consequence the (annealed average of the) logarithm of the number of stable states coincides with $S\left(Q_{0}\right)$. When we inject $Q_{0}$ into (37) the first two lines can be easily computed and read:

$$
-\lambda\left(r-r_{c}\right)+\frac{p}{4} d q^{p-1}+\frac{p}{2 T}\left(r-r_{c}\right)\left(1-q^{p-1}\right)+\frac{p(p-1)}{4}\left(r-r_{c}\right)^{2} q^{p-2}
$$

whereas the computation of the third one, which reduces only to $\operatorname{Tr} \ln M / 2$, is slightly more subtle. Since the operator (38) is diagonal in Fourier space, we get:

$$
\frac{1}{2} \operatorname{Tr} \ln M=\frac{1}{2} \ln \left(r^{2}-q d\right)+\frac{1}{2} \sum_{\omega \neq 0} \ln \left(\begin{array}{cc}
\hat{R}(\omega) & \hat{C}(\omega) \\
0 & \hat{R}^{*}(\omega)
\end{array}\right),
$$

where the Fourier transform of a function $F(\tau)$ is defined as:

$$
\hat{F}(\omega)=\int_{0}^{t^{*}} e^{-i \omega \tau} F(\tau) d \tau \quad, \quad \omega=\frac{2 \pi n}{t^{*}} \quad n=0, \pm 1, \pm 2, \ldots
$$

The function $R_{c}\left(t, t^{\prime}\right)$ is causal and the associated operator is upper triangular with diagonal elements equal to unity. Its determinant hence is one (here the Ito convention is crucial), as it should because it does not give any contribution to the action in the standard case. As a consequence we have:

$$
\frac{1}{2} \sum_{\omega \neq 0} \ln \left(\begin{array}{cc}
\hat{R}(\omega) & \hat{C}(\omega) \\
0 & \hat{R}^{*}(\omega)
\end{array}\right)=-\ln \left(r_{c}\right) \quad,
$$

Collecting all the pieces together and using the equation on $q, r, r_{c}$ and $d$ obtained in section $\mathrm{VA}$ we find that the action $S\left(Q_{0}\right)$ reads:

$$
\begin{aligned}
S\left(Q_{0}\right) / N & =\frac{1}{2}(1+\ln p-\ln 2)-\mathcal{E}^{2}+\frac{1}{2}\left(\frac{\mathcal{E}-\sqrt{\mathcal{E}^{2}-\mathcal{E}_{c}^{2}}}{\mathcal{E}_{c}}\right)^{2}+\ln \left(-\mathcal{E}-\sqrt{\mathcal{E}^{2}-\mathcal{E}_{c}^{2}}\right) \\
\mathcal{E}_{c} & =-\sqrt{\frac{2(p-1)}{p}}
\end{aligned}
$$

As expected, this expression coincides with the logarithm of the number of TAP states computed by Crisanti and Sommers 13 .

Note that this formula is correct only for $\mathcal{E}<\mathcal{E}_{c}$. For $\mathcal{E}>\mathcal{E}_{c}$ the formalism tells us that there are no stable states as follows: in this energy regime $D$ and $\hat{\lambda}$ remain of order one even for infinite $t^{*}$ and the action acquires a negative contribution of order $t^{*}$. This is as it should: since for these energy values there are only metastable states with finite life-times, the longer we set $t^{*}$ the less metastable states we find.

In general for finite dimensional glassy systems the interesting quantity will be the logarithm of the number of metastable states with finite lifetime, which can be obtained plugging the solution $Q(\tau)$ into the action $\mathrm{S}$ for a finite value of $t^{*}$. In the following we compute this quantity at zero temperature.

\section{The zero temperature case}

At zero temperature the equation (40) is particularly simple. Introducing the notation $R(\tau)=R_{c}(\tau)+\left(r-r_{c}\right) / t^{*}$ we find that the equation on the Fourier transform of $R_{c}$ reads:

$$
-i \omega \hat{R}_{c}(\omega)-\lambda \hat{R}_{c}(\omega)+\frac{p(p-1)}{2} \hat{R}_{c}(\omega) \hat{R}_{c}(\omega)+1=0
$$

For each frequency there are two solutions: 


$$
\hat{R}_{c}^{ \pm}(\omega)=\frac{1}{p(p-1)}\left(-\Lambda \pm \sqrt{\Lambda^{2}-2 p(p-1)}\right), \quad \Lambda=\lambda+i \omega
$$

In the following we focus on the two solutions $R_{c}^{+}(\tau)$ and $R_{c}^{-}(\tau)$ which correspond respectively to taking the Fourier transform of $\hat{R}_{c}^{+}(\omega)$ and $\hat{R}_{c}^{-}(\omega)$. Using that at zero temperature $C(\tau)=1$ one can decompose $S(Q)$ in two terms such that all the dependence on $t^{*}$ is contained only in one of them:

$$
\begin{aligned}
S / N\left(Q^{ \pm}\right) & =\frac{1}{2}(1-\ln p-\ln 2)-\mathcal{E}^{2}+\frac{1}{2}\left(\frac{\mathcal{E} \mp \sqrt{\mathcal{E}^{2}-\mathcal{E}_{c}^{2}}}{\mathcal{E}_{c}}\right)^{2}+\ln \left(-\mathcal{E} \mp \sqrt{\mathcal{E}^{2}-\mathcal{E}_{c}^{2}}\right) \\
& -\frac{p(p-1)}{4} \int_{0}^{t^{*}} R_{c}^{ \pm}(\tau) R_{c}^{ \pm}(-\tau) d \tau+\frac{1}{2} \sum_{\omega} \ln \left(\hat{R}_{c}^{ \pm}(\omega) \hat{R}_{c}^{ \pm}(-\omega)\right)
\end{aligned}
$$

The computation of the second line is performed in the Appendix B. It turns out that, as in the static case [12], the dominant contribution is given by $R_{c}^{-}$for $\mathcal{E}_{R S B}<\mathcal{E}<\mathcal{E}_{c}$, by $R_{c}^{+}$for $-\mathcal{E}_{c}<\mathcal{E}<-\mathcal{E}_{R S B}$ and for $-\mathcal{E}_{c}<\mathcal{E}<-\mathcal{E}_{c}$ the two saddle point contributions are the same (see Appendix B). Note that at zero temperature, $\mathcal{E}$ is the energy density of the system. The final result is

$$
\begin{aligned}
S(Q) & =\operatorname{Re}\left(\frac{1}{2}(1-\ln p-\ln 2)-\mathcal{E}^{2}+\frac{1}{2}\left(\frac{\mathcal{E} \mp \sqrt{\mathcal{E}^{2}-\mathcal{E}_{c}^{2}}}{\mathcal{E}_{c}}\right)^{2}+\ln \left(-\mathcal{E} \mp \sqrt{\mathcal{E}^{2}-\mathcal{E}_{c}^{2}}\right)\right) \\
& -\int d \omega \rho_{p}(\omega+p \mathcal{E}) \ln \left(1-\exp \left(-t^{*}|\omega|\right)\right)+t^{*} \int_{-\infty}^{0} d \omega \rho_{p}(\omega+p \mathcal{E}) \omega
\end{aligned}
$$

where $\rho_{p}(x)=\sqrt{2 p(p-1)-x^{2}} /(\pi p(p-1))$ is the Wigner semi-circle law. In fig. 1 we plot (68) for $p=3$ and different values of $t^{*}$ as a function of the energy density $\mathcal{E}$. For very large values of $t^{*}, S(Q)$ converges to the logarithm of the number of stable states. Note that for a finite dimensional system we expect a similar behavior but with a vanishing curve for infinite $t^{*}$. Finally, we remark that the formula (68) has a simple interpretation. In fact the first line coicides with the number of saddles with energy density $E$. Moreover, since the spectrum of the Fokker-Planck operator for an harmonic oscillator with frequency $\omega$ is [33] $E_{n}=(n+1 / 2)|\omega|-\omega / 2$, the second line of (68) corresponds to the contribution due to a collection of harmonic oscillators with frequency distributed by the semicircle law centered in $-p \mathcal{E}$. This distribution is exactly the same of the eigenvalues of the energy Hessian evaluated in saddles with energy density $\mathcal{E}$ [12]. As a consequence, at zero temperature, the spectrum of the Fokker-Planck operator for the p-spin spherical model coincides with the one obtained making an harmonic expansion around each saddle (also the instable ones).

\section{Time dependent configurational entropy}

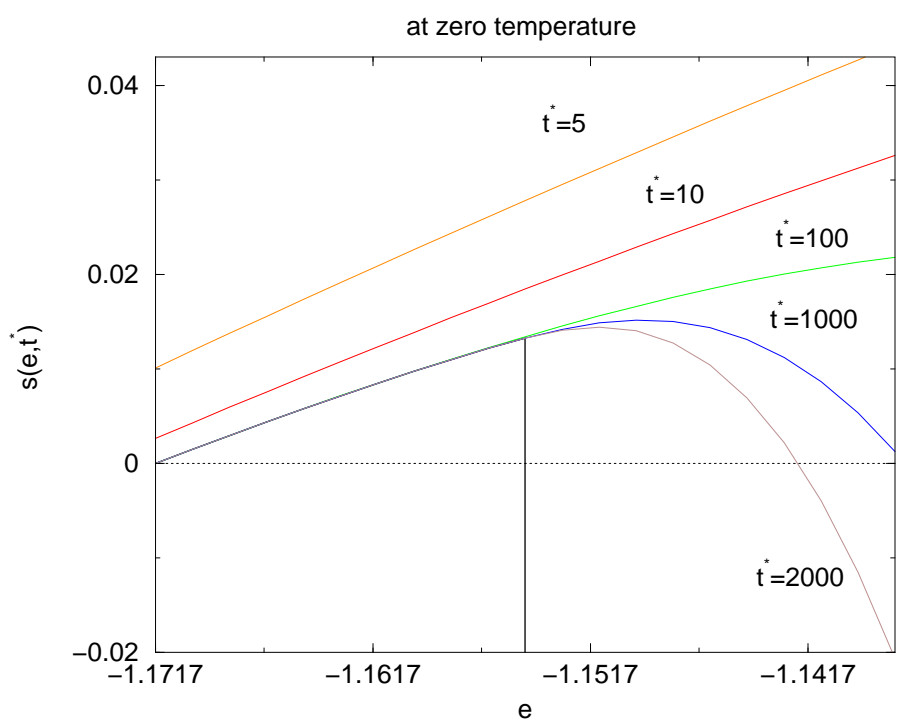

FIG. 4. Time dependent configurational entropy $s\left(e, t^{*}\right)$ at $T \rightarrow 0$ for $p=3$ as a function of the energy density $e$ and of the time $t^{*}$. From top to bottom: $t^{*}=5,10,100,1000,2000,+\infty$. Note the infinite time limit curve with positive configurational entropy a mean-field artifact. 


\section{THE TWO-GROUPS ANSATZ AND SUPERSYMMETRY BREAKING}

Twenty years ago, when people started to search for replica symmetry breaking solutions of the SherrigtonKirkpatrick model, Bray and Moore [35] proposed a two-groups Ansatz for the famous $Q_{a, b}$ matrix [3]. At a first sight the results seemed a little bit strange since the $\lim _{n \rightarrow 0} \overline{Z^{n}} \neq 1 !(-$ means the average over disorder). It turned out [36] that in the limit of $n$ going to zero, the logarithm of $\overline{Z^{n}}$ equals the the logarithm of the number of TAP states, i.e. the long-time limit of the configurational entropy (25). The reason of this "coincidence" has been completely obscure until now. For instance, for many mean-field spin glass models the complexity was computed starting from TAP states and, after, it was checked that the two-groups Ansatz gave back the correct result [36, 37, 28].

Using the properties of the dynamical solutions presented in previous sections we can unveil why the Bray and Moore Ansatz allows one to calculate the long-time limit of the configurational entropy (25). In fact this Ansatz is isomorphic to zero frequency part of the dynamical calculation for $t^{*} \rightarrow \infty$. This can be shown by the supersymmetric formalism for Langevin dynamics 33, 38]. Within this framework all the two points correlation functions between fields $S_{i}(t)$ and $\hat{S}_{i}(t)$ can be encoded in:

$$
\begin{aligned}
Q(1,2) & =\sum_{i=1}^{N}\left\langle S_{i}(1) S_{i}(2)\right\rangle \quad 1=\left(T t_{1}, \bar{\theta}_{1}, \theta_{1}\right) \\
S_{i}(1) & =S_{i}\left(t_{1}\right)+\bar{\theta}_{1} \theta_{1} \hat{S}_{i}(t)+\bar{c}_{i}\left(t_{1}\right) \theta_{1}+\bar{\theta}_{1} c_{i}\left(t_{1}\right)
\end{aligned}
$$

where $\bar{\theta}_{1}, \theta_{1}$ are Grassmann variables and $\bar{c}_{i}(t), c_{i}(t)$ are fermion fields [33, 38]. Using this formalism the dynamical solution (58) giving back the configurational entropy reads at large times:

$$
\begin{aligned}
Q(1,2) & =Q_{c}(1,2)+q+\left(\bar{\theta}_{1} \theta_{1}+\bar{\theta}_{2} \theta_{2}\right) \frac{r-r_{c}}{t^{*}}+\bar{\theta}_{1} \theta_{1} \bar{\theta}_{2} \theta_{2} \frac{d}{\left(t^{*}\right)^{2}} \\
Q_{c}(1,2) & =\left(1+\frac{1}{2}\left(\bar{\theta}_{1}-\bar{\theta}_{2}\right)\left[\theta_{1}+\theta_{2}-\left(\theta_{1}-\theta_{2}\right) \operatorname{sign}\left(t_{1}-t_{2}\right)\right] \frac{1}{T} \frac{\partial}{\partial t_{1}}\right)\left(C_{c}\left(t_{1}-t_{2}\right)-q\right)
\end{aligned}
$$

The function $Q_{c}(1,2)$ is supersymmetric, whereas the last two terms in the right hand side of (71) break supersymmetry.

On the other hand, the two-groups Ansatz consists in a symmetric $Q_{a, b}$ matrix:

$$
\begin{array}{ll}
Q_{a, b}=1+\frac{B}{m} \quad a=b \leq m, \quad Q_{a, b}=1-\frac{B}{m} \quad m<a=b \leq n \\
Q_{a, b}=A+\frac{B}{m} \quad a \neq b ; \quad a, b \leq m \\
Q_{a, b}=A-\frac{B}{m} \quad a \neq b ; \quad m<a, b \leq n \\
Q_{a, b}=A-\frac{C}{m^{2}} \quad a \leq m, \quad m<b \leq n
\end{array}
$$

where one has to take the $m \rightarrow \infty$ and $n \rightarrow 0$ limits. The functional dependence of the dynamical and replica free energy of, respectively, $Q(1,2)$ and $Q_{a, b}$ is the same [38]. Indeed, the kinetic term in the dynamical free energy, which does not have a correspondent in the static case, is zero for the dynamical solution (71). Moreover if one puts $B=\left(r-r_{c}\right) T, 2 C=T^{2} d, A=q$ the two matrices $Q(1,2)$ and $Q_{a, b}$ lead to the same results under tracing, convolution and term by term product. For instance, one can easily obtain:

$$
\begin{aligned}
\sum_{a} f\left(Q_{a, a}\right) & =\int d 1 f(Q(1,1))=2 B \\
\sum_{a, b} f\left(Q_{a, b}\right) & =\int d 1 d 2 f(Q(1,2))=2\left(f^{\prime}(1)-f^{\prime}(A)\right) B+f^{\prime \prime}(A) B^{2}+2 f^{\prime}(A) C \\
\operatorname{Tr}_{a, b} \log Q & =\operatorname{Tr}_{1,2} \log Q=-2 \log (1-A)+\log \left((1-A+B)^{2}-2 A C\right)
\end{aligned}
$$

As a consequence the computation, which make use of the two-groups Ansatz, is isomorphic to the dynamical one for $t \rightarrow t^{*}$. Therefore the replica symmetry breaking scheme encoded in this Ansatz can be finally understood: it is a way to implement the dynamical computation in a replica formalism. There are, however, two important differences 
between the two approaches. First of all, in the dynamical computation we are not free to choose between different Ansätze the one which gives back the long-time limit of the configurational entropy (25), but we have simply to solve the equations of motion. This clearly makes the procedure inambiguous, unlike the case of the replica computation. Moreover the two approaches lead to the same results only if a dynamical solution with the correct values of $q, d, r$ exists. It could then happen (cf. the discussion on the configurational entropy of the SK model) that the equations on $q, d, r$ admit a solution but there is no dynamical solution corresponding to these values. As a consequence even if the static computation, i.e. the sum over all TAP solutions or the computation by the two-groups scheme, predicts the existence of an exponential number of stable states the more correct dynamical calculation does not.

\section{CONCLUSIONS}

In this work we have shown how to put the questions related to metastable states in glasses in a manner valid for finite-dimensional systems.

We have used the construction of Gaveau and Schulman to define the metastable states. This construction requires the existence of a 'gap' in the lifetime, so that one can associate 'states' with distributions that are stable for much longer than a given time $t^{*}$, and 'transient processes' those that decay much faster. There is no such gap in real glasses, so our use of this construction has to be considered partly as a definition inspired in the cases where there is.

A reasonable criterion for the relevance of any quantity will hence be that they are not too sensitive to the exact value chosen for $t^{*}$. For example, if we consider a temperature associated with the $\alpha$-relaxation as $T_{t^{*}}$ with $t^{*} \lesssim t_{\alpha}$, this definition is meaningful to the extent that it is stable with respect to a change in $t^{*}$ of, say, an order of magnitude.

Next, there is the question as to whether $T_{t^{*}}$ indeed reproduces the fluctuation-dissipation temperature. This and other results depend on the validity of the flatness hypotheses à la Edwards (for which positive evidence begins to appear [39]). In this paper we have formulated the hypothesis in a manner applicable to positive temperatures and finite dimensions (as well as to vibrated systems) - but we have not attempted to prove it. It may be, however, that writing it in the form (27), can be a good starting point for doing this. Moreover, the form (27) (and (28)) lends itself naturally to a generalisation to cases in which a system has more than two widely separated timescales and temperatures.

Finally, the computation in section $\mathrm{V}$ has allowed us to check the mean-field results without relying on the TAP states, themselves an intrinsically mean-field concept. The kind of solutions that dominate break causality and have positive action, but satisfy time-reversal and a non-causal form of FDT. Unlike the barrier-crossing solution of Lopatin and Ioffe [40], they have in this sense only some of the properties of true instantons. Moreover, the dynamical computation unveils the meaning of the two-groups Ansatz [36], which allows one to compute the number of stable states within a replica formalism.

\section{Acknowledgments}

We wish to thank L. Ioffe, J. L. Lebowitz and L. S. Schulman for useful suggestions. 


\section{APPENDIX A. PURE BARRIERS.}

Let us show how to define 'pure barriers' using a supersymmetric extention of the Fokker-Planck operator.

For a system with $N$ degrees of freedom, introduce the $N$ fermion creation and annihilation operators $a_{i}$ and $a_{i}^{\dagger}$, with anticommutation relations $\left[a_{i}, a_{j}^{\dagger}\right]_{+}=\delta_{i j}$. Define the supersymmetric charges as:

$$
\bar{Q}=\left(T p_{i}-i E_{, i}\right) a_{i}^{\dagger} ; Q=p_{i} a_{i}
$$

The supersymmetric operator $H_{S U S Y}$

$$
H_{S U S Y}=[\bar{Q}, Q]_{+}=H+\frac{\partial^{2} E}{\partial x_{i} \partial x_{j}} a_{j}^{\dagger} a_{i}
$$

commutes with the charges, and with the fermion number operator. In the zero-fermion subspace it coincides with the Fokker-Planck operator.

Applying the operator $\bar{Q}$ to all but the lowest Fokker-Planck (zero-fermion) eigenvectors, one obtains a one-fermion eigenvector. Going back to the example of low-temperature dynamics in a potential of section III, one can see that the lowest one-fermion eigenvectors correspond to distributions associated to the barriers. Indeed, one can convince oneself that in a low temperature multi-dimensional system, the lowest eigenvectors with $k$ fermions correspond to barriers with $k$ unstable directions (see Ref. [41], where these questions are discussed in detail, and used to derive Morse theory results).

Now, by analogy with the argument motivating the construction of pure states, it is reasonable to define 'barrier distributions' in general as the lowest eigenvectors of $H_{S U S Y}$ with lowest eigenvalues in subspaces with $k$ fermions. This definition will make sense whenever there is a gap in the spectrum, whatever the origin of such a gap (large $N$, low $T$, etc). One can then use traces of $e^{-t^{*} H_{S U S Y}}$ to calculate expectation values. 


\section{APPENDIX B.}

The aim of this section is to calculate the last line of (67). Instead of making the computation by brute force, we will use the exact results that can be obtained in the $p=2$ case. For $p=2$ the spherical model is a simple collection of harmonic oscillators with frequency distributed with a semicircle law centered around the value of the spherical multiplier. The spectrum of the Fokker-Planck operator for an harmonic oscillator with frequency $\omega$ is [33: $E_{n}=(n+1 / 2)|\omega|-\omega / 2$. Therefore for $p=2$ and at zero temperature, the logarithm of the trace of the evolution operator reads:

$$
\overline{\ln \left(T r e^{-t^{*} H}\right)}=-N \int d \omega \rho(\omega+2 \mathcal{E}) \ln \left(1-\exp \left(-t^{*}|\omega|\right)\right)+N \int_{-\infty}^{0} d \omega \rho(\omega+2 \mathcal{E}) t^{*} \omega
$$

where $\rho(x)$ is the Wigner semicircle law. One can obtain this result also by the functional formalism. In the computation of section $(\overline{\mathrm{VC}})$ we obtained an action which for $p=2$ reads:

$$
\begin{aligned}
S / N= & \frac{1}{2}-\mathcal{E}^{2}+\frac{1}{2}\left(\mathcal{E} \mp \sqrt{\mathcal{E}^{2}-1}\right)^{2}+\ln \left(-\mathcal{E} \mp \sqrt{\mathcal{E}^{2}-1}\right) \\
& -\frac{1}{2} \sum_{n} R^{ \pm}(n) R^{ \pm}(n)+\frac{1}{2} \sum_{n} \ln \left(R^{ \pm}(n) R^{ \pm}(-n)\right)
\end{aligned}
$$

where we have written $R(t)$ instead $R_{c}(t)$ since $r=r_{c}$ for $p=2$. This is directly related to the fact that there is no configurational entropy for $p=2$ and therefore the two-time functions relax asymptotically faster than $1 / t^{*}$. For $\mathcal{E}<-1$ the $p=2$ model is a collection of stable harmonic oscillators. One can easily compute $R_{c}(t)$ starting from the result for a single oscillator and integrating over the Wigner distribution. As expected, this function coincides with $R_{c}^{-}(t)$. As a consequence for $\mathcal{E}<-1$ we expect that $\overline{\ln \operatorname{Tr} e^{-t^{*} H}}=N S\left(Q^{-}\right)$. On the other hand for $\mathcal{E}>1$ the $p=2$ model is a collection of unstable harmonic oscillators which can mapped to the previous case changing the sign of each $\omega$. As a consequence for $\mathcal{E}>1$ we expect that $\overline{\ln \operatorname{Tr} e^{-t^{*} H}}=N S\left(Q^{+}\right)$. In the intermediate energy regime a priori one has to consider both solutions. Since the functional computation should give back the result (82), the last line of (83) reads:

$$
\begin{aligned}
& -\frac{1}{2} \sum_{n} R^{ \pm}(n) R^{ \pm}(n)+\frac{1}{2} \sum_{n} \ln \left(R^{ \pm}(n) R^{ \pm}(-n)\right)= \\
= & -\int d \omega \rho(\omega+2 \mathcal{E})\left(-t^{*} \frac{\omega}{2}+\ln \left|e^{\omega t^{*} / 2}-e^{-\omega t^{*} / 2}\right|\right) \pm i \pi \int_{-\infty}^{0} d \omega \rho(\omega+2 \mathcal{E})
\end{aligned}
$$

if the determination of the logarithm in the first line of $(83)$ is such that $\ln -1=-i \pi$. Note that for $\mathcal{E}<-1$ all the oscillators are stable, as a consequence the last term in (84) vanishes and (83) coincides with (82). For $\mathcal{E}>1$ all the oscillators are unstable, as a consequence the last term in (84) equals $i \pi$ and cancels the $-i \pi$ coming from the first logarithm in 83 , and one obtains the same results that for $\mathcal{E}<-1$ with an additional term $\int d \omega \rho(\omega+2 \mathcal{E}) t^{*} \omega$. Finally, in the intermediate energy regime the first line in (83) is complex and its imaginary part cancels exactly the imaginary contribution coming from the last term in (84). In this case the the two saddle point contributions are the same, therefore to obtain (82) one can consider only one of them. However to obtain the expected value of $R(t)$ one has to sum on saddle points.

For $p$ greater than two, the equation on $R_{c}$ has the same form of (40) but for a $p=2$ spherical model at zero temperature with a variance of the couplings $J^{2}=p(p-1) / 2$. As a consequence the last line of (67) reads:

$$
-\int d \omega \rho(\omega+p \mathcal{E})\left(-t^{*} \frac{\omega}{2}+\ln \left|e^{\omega t^{*} / 2}-e^{-\omega t^{*} / 2}\right|\right) \pm i \pi \int_{-\infty}^{0} d \omega \rho(\omega+p \mathcal{E})
$$

[1] B. Gaveau and L.S. Schulman, Jour. Math. Phys 39 (1998) 1517.

See also:

B. Gaveau and L.S. Schulman, Jour. Math. Phys 37 (1996) 3897; Phys. Lett. A229 347;

B. Gaveau, A. Lesne and L.S. Schulman, Phys. Lett. A258 (1999) 222. 
[2] S. F. Edwards, in Granular Matter: An Interdisciplinary Approach, A. Mehta ed. Springer (1994), and references therein. See also: A. Mehta, R. J. Needs and S. Dattagupta; Journal of Statistical Physics 681131 (1992) and: R. Monasson and O. Pouliquen, Physica A 236395 (1997).

[3] M. Mézard, G. Parisi and M. Virasoro, Spin Glass Theory and Beyond (1987) (Singapore: World Scientific).

[4] J-P Bouchaud, L. F. Cugliandolo, J. Kurchan and M. Mézard cond-mat/9702070; in Spin-glasses and random fields, A. P. Young ed. (World Scientific, Singapore).

[5] J. Kurchan, in : Jamming and Rheology: Constrained Dynamics on Microscopic and Macroscopic Scales (1997), http://www.itp.ucsb.edu/online/jamming2/, and S. F. Edwards, A. Liu and R.S. Nagel Eds., to be published

J. Kurchan, cond-mat/9909306,J. Phys. Condensed Matter, to be published .

[6] S. Franz and M. A. Virasoro, J. Phys. A 33891 (2000).

[7] T. R. Kirkpatrick and D. Thirumalai, Phys. Rev. B 36, 5388 (1987), ibid 37, 5342 (1988), Phys. Rev. A 37, 4439 (1988). D. Thirumalai and T. R. Kirkpatrick, Phys. Rev. B 38, 4881 (1988). T. R. Kirkpatrick and D. Thirumalai, J. Phys. A 22, L149 (1989). T. R. Kirkpatrick and P. Wolynes, Phys. Rev. A 35, 3072 (1987), Phys. Rev. B 36, 8552 (1987).

T. R. Kirkpatrick, D. Thirumalai, P. G. Wolynes, Phys. Rev. A 40, 1045 (1989).

[8] M. Mézard and G. Parisi, phys. Rev. Lett. 82 747 M. Mézard and G. Parisi, J. Phys. Chem. 111 1076 (1999); M. Mézard, First Steps in Glass Theory cond-mat/000517.

[9] D. J. Thouless, P. W. Anderson, and R. G. Palmer, Phil. Mag. 35, 593 (1977)

H. Rieger, Phys. Rev. B 46, 14655 (1992)

J. Kurchan, G. Parisi, and M. A. Virasoro, J. Phys. I France 3, 1819 (1993).

[10] C. De Dominicis, Phys. Rep. B 67, 37 (1980).

[11] A. Bray and M. Moore, J. Phys. C13 (1980) L469

[12] A. Cavagna, I. Giardina and G. Parisi, Phys. Rev. B 57, 11251 (1998).

[13] A. Crisanti and H.-J. Sommers, J. Phys. I (France) 5, 805 (1995).

[14] A. Barrat, R. Burioni, and M. Mézard, J. Phys. A 29, L81 (1996),

S. Franz and G. Parisi, J. Phys. I (France) 5, 1401 (1995).

[15] G. Biroli, J. Phys. A 32 (1999) 8365

[16] V.S. Dotsenko, M.V. Feigel'man and L.B. Ioffe, Spin-glasses and related problems, Soviet Scientific Reviews, vol.15 (Harwood, 1990).

[17] L. F. Cugliandolo and J. Kurchan, Phys. Rev. Lett. 71, 173 (1993). Phil. Mag. B 71, 501 (1995).

[18] L.F. Cugliandolo, J.Kurchan and L.Peliti, Phys. Rev. E 55, 3898 (1997).

[19] R. Monasson, Phys. Rev. Lett. 75, 2847 (1995).

[20] M. A. Virasoro, unpublished.

[21] L.L. Bonilla, F. G. Padilla and F. Ritort, Physica A250 (1998) 315.

[22] Th. M. Nieuwenhuizen, Phys. Rev. E 61267 (2000).

[23] G. Parisi, Phys. Rev. Lett. 79, 3660 (1997)

[24] W. Kob and J.-L. Barrat, Eur. Phys. Lett. 46, 637 (1999)

[25] M. Sellitto, Eur. J. Phys. B 4, 135 (1998).

[26] R. Di Leonardo, L. Angelani, G. Parisi and G. Ruocco, cond-mat/000131]

[27] P.H. Stillinger and T.A. Weber Phys. Rev. A 25978 (1982). P.H. Stillinger and T.A. Weber, Science 225, 983 (1984). B. Coluzzi, G. Parisi and P. Verrocchio Phys. Rev. Lett. 84306 (2000)

F. Sciortino, W. Kob, and P. Tartaglia, Phys. Rev. Lett. 833214 (1999), W. Kob, F. Sciortino, and P. Tartaglia, Europhys. Lett. 495906 (2000).

A. Crisanti and F. Ritort, Activated processes and Inherent Structure dynamics of finite-size mean-field models for glass, cond-mat/9911226, Potential energy landscape of simple p-spin models for glasses, cond-mat/9907499

[28] G. Biroli and R. Monasson, Europhys. Lett. 50 (2000) 155.

[29] See: R. Melin, J. Physique I 6 (1996) 469; R. Melin and B. Butaud, J. Physique I 7 (1997) 691.

[30] T.W. Ruijgrok and J.A. Tjon, Physica 65539 (1973);

G. Biroli and R. Monasson, J. Phys. A 31 L391 (1998).

[31] This follows from the fact that, for a Fokker-Planck process: $\left\langle x\left|e^{-t^{*} H}\right| x\right\rangle \sim\left(4 \pi T t^{*}\right)^{-\frac{1}{2}}$ as $t^{*} \rightarrow 0$, a constant independent of the coordinates.

[32] In the thermodynamic limit it is in fact more proper to compare, instead of $t^{*}$, the timescale given by $N\left[\langle\langle H\rangle\rangle_{t^{*}}\left(E_{o}\right)\right]^{-1}=$ $-\left(\frac{1}{N} \frac{\partial \mathcal{S}_{t^{*}}\left(E_{0}\right)}{\partial t^{*}}\right)^{-1}$ (i.e. inverse average eigenvalue of the Fokker-Planck operator), which is the conjugate variable to $t^{*}$ under Legendre transformation.

[33] J. Zinn-Justin, Quantum Field Theory and Critical Phenomena, Clarendon Press 1997.

[34] We should take averages over the logarithm of the trace. In this kind of models, it seems safe to take instead the (annealed) average over the trace itself 13.

[35] A.J. Bray and M. Moore, Phys. Rev. Lett. (1978), 411068

[36] A.J. Bray and M. Moore, J. Phys. C 13 (1980), L907

[37] G. Parisi and M. Potters, Eur. Phys. Lett. 32 (1995) 13.

[38] J. Kurchan, J. Phys. France 2, 1333 (1992); S. Franz and J. Kurchan, Europhys. Lett. 20, 197 (1992). 
[39] A. Barrat, J. Kurchan, V. Loreto and M. Sellitto, cond-mat/000614d.

[40] L. B. Ioffe and D. Sherrington, Phys. Rev. B 57, 7666 (1998). A. V. Lopatin and L. B. Ioffe, Phys. Rev. B 60, 6412 (1999); cond-mat/9907135.

[41] E. Witten, J. Diff. Geom 17, (1982) 661. 